\title{
Structural reliability analysis using response surface method with improved genetic algorithm
}

\author{
Yongfeng Fang ${ }^{* 1}$ and Kong Fah Tee ${ }^{2 a}$ \\ ${ }^{1}$ School of Mechanical Engineering, Guizhou University of Science Engineering, Bijie 551700, China \\ ${ }^{2}$ Department of Engineering Science, University of Greenwich, Kent ME4 4TB, United Kingdom
}

\begin{abstract}
For the conventional computational methods for structural reliability analysis, the common limitations are long computational time, large number of iteration and low accuracy. Thus, a new novel method for structural reliability analysis has been proposed in this paper based on response surface method incorporated with an improved genetic algorithm. The genetic algorithm is first improved from the conventional genetic algorithm. Then, it is used to produce the response surface and the structural reliability is finally computed using the proposed method. The proposed method can be used to compute structural reliability easily whether the limit state function is explicit or implicit. It has been verified by two practical engineering cases that the algorithm is simple, robust, high accuracy and fast computation.
\end{abstract}

Keywords: structural reliability; response surface method; improved genetic algorithm

\section{Introduction}

The response surface method (RSM) is a very important method for structural reliability analysis. The main advantages of RSM are because it is not restrained by the number of random variables and it is also not limited by the form of the limit state function whether it is implicit or explicit (Ellingwood and Galambos 1982, Mahmoodian et al. 2012, Jiang et al. 2012, Fang et al. 2014, Tee et al. 2015). In addition, the RSM is simple to perform with high accuracy (Bai et al. 2014, Fang et al. 2013, Su et al. 2015). Nevertheless, if the initial point of choice in the RSM is unreasonable and the objective function is highly nonlinear, then the rate of convergence of the RSM is slow. Therefore, the RSM for structural reliability estimation in a wide range of applications has been limited.

An improved RSM has been developed for structural reliability evaluation ( $\mathrm{Li}$ and Chen 2013). In this method, the rate of convergence of the RSM is accelerated by rotating the coordinate system to compute the structural reliability. However, due to the rotation of the coordinate system in each computation step, the total computational time apparently has been increased when there are many random variables. Another improved RSM for reliability analysis of structures has also been proposed by Kang et al. (2010). Although the precision efficiency of the proposed improved RSM method is higher, its weight function has to be determined in structural reliability estimation. The convergence may not be guaranteed if the weight function

\footnotetext{
*Corresponding author, Ph.D.

E-mail: fangyf_9707@126.com

${ }^{\mathrm{a}} \mathrm{Ph} . \mathrm{D}$.
}

E-mail: K.F.Tee@gre.ac.uk is not correctly determined. A new quadratic function has been constructed to substitute the RSM, some variables are chosen to compute the structural reliability (Basaga et al. 2012). The rate of convergence is higher, but the proposed method is complex. The precision and accuracy of the proposed method is influenced by the complexity.

The genetic algorithm (GA) has been considered in computer applications since 1960s. Its technological merits are high degree of parallelism, less dependent on the initial value and superior robustness in the computation of extremum (Xuan and Chen 2000, Michhalewicz 1994, Yang et al. 2002). The GA has been used to study structural optimization (Shao et al. 2001, Tee et al. 2014, Khan and Tee 2016). It has been shown that soft computing is a superior method for structural reliability computation, but the study is relatively simple. An improved genetic algorithm has been proposed by He and Liang (2001). The method has accelerated convergence and improved the computational efficiency by changing trends of search points based on the improved gradient. The structural optimization has been studied by using cuckoo search algorithm and GA (Ponnambalam 2014). It has been shown that the proposed approach is superior when it is applied to discrete variables.

Based on the above literature review, the RSM for structural reliability computation is studied in this paper using the improved gradient of GA. The method is suitable to be incorporated with the response surface function for both explicit and implicit form. It has been verified by two examples that the proposed method can be used to compute structural reliability with fast convergence and high computational efficiency.

\section{Response Surface Method (RSM)}


The structural limit state function (LSF) $G(X)=g\left(x_{1}, x_{2}, \ldots, x_{n}\right)$ in the RSM can be formulated using a quadratic polynomial function as follows.

$$
Z=g(\mathbf{x})=a+\sum_{i=1}^{n} b_{i} x_{i}+\sum_{i=1}^{n} c_{i} x_{i}^{2}
$$

where $x_{i}(i=1,2, \ldots, n)$ is space variable of the LSF and $a, b_{i}$, $c_{i}(\mathrm{i}=1,2, \ldots, n)$ are the coefficients of Eq. (1).

The computation procedure of structural reliability using the RSM is given as follows.

The mean point of the random variables is chosen as the initial point $X^{1}=\left[x_{1}^{1}, x_{2}^{1}, \cdots, x_{n}^{1}\right]$

$g\left(x_{1}^{1}, x_{2}^{1}, \cdots, x_{n}^{1}\right) \quad$ and $\quad g\left(x_{1}^{1}, x_{2}^{1}, \cdots, x_{i}^{1} \pm f \sigma_{i}, \cdots, x_{n}^{1}\right) \quad$ will be determined, $f$ is assigned to 3 in the first step computation and 1 in the next step. The $2 n+1$ value will be obtained and the value will be used in the coefficients of the Eq. (1) to obtain the RSM. The reliability index $\beta^{k}$ and its design test point $X_{D}^{k}$ of the structure will be determined by using the first order second moment method (Fang et al. 2015) based on the response surface function ( $k$ is the number of the iteration).

If Eq. (2) can be established, then it is possible to evaluate structural reliability.

$$
\left\|\beta^{k}-\beta^{k-1}\right\|<\varepsilon
$$

where $\beta^{k}$ is the reliability index of the structure. On the other hand, if Eq. (2) cannot be established, then the new design testing point $X_{M}^{k}$ will be obtained as follows.

$$
X_{M}^{k}=X^{k}+\left(X_{D}^{k}-X^{k}\right) \frac{g\left(X^{k}\right)}{g\left(X^{k}\right)-g\left(X_{D}^{k}\right)}
$$

where $X^{k}$ is the initial point where the response surface function can be computed by $k$ th iteration. $X_{D}^{k}$ and $X_{M}^{k}$ are the design testing point and the interpolation point at the $k$ th response surface function, respectively. $g\left(X^{k}\right)$ and $g\left(X_{D}^{k}\right)$ are the values of the LSF which are corresponding to $X^{k}$ and $X_{D}^{k}$, respectively.

A huge amount of computational time and bigger error will be produced by using the above method if the LSF is a non-linear performance function. In addition, when the higher accuracy is required, the number of interpolation and the order of the approximation function will be increased. It has been shown that the computation becomes more difficult and complex. Thus, a new method described in Section 3 has been proposed to overcome the problem.

\section{Improved GA by fitness function}

In structural reliability estimation, the LSF can be transformed into the fitness function (FF) as follows.

$$
F(X)= \begin{cases}c_{\max }-G(X) & \text { if } G(X)<c_{\max } \\ 0 & \text { otherwise }\end{cases}
$$

where $c_{\max }$ is a constant which is the maximum value of $G(X)$ in the evolutionary process.

If the LSF is explicit, differentiable and continuous function, the new FF can be established by using the gradient of the LSF for accelerating the convergence in the evolutionary process. The new FF is given as follows.

$$
\begin{aligned}
& F^{\prime}(X)=\alpha \cdot \frac{F(X)-F_{\text {min }}(Z)}{F_{\text {max }}(Y)-F_{\text {min }}(Z)}+ \\
& (1-\alpha) \frac{\left\|\nabla F(X)-\nabla F_{\text {min }}(Z)\right\|}{\left\|\nabla F_{\text {max }}(Y)-\nabla F_{\text {min }}(Z)\right\|}
\end{aligned}
$$

where $\alpha \in[0,1]$ is power factor and it is determined according to one's experience. $F_{\max }(Y)$ and $F_{\min }(Z)$ are the maximum value of the individual fitness in the current generation and the minimum value of the individual fitness in the previous generation, $\nabla F(X)$ is the gradient of the LSF, $\nabla F_{\max }(Y)$ and $\nabla F_{\min }(Z)$ can be determined by using Eq. (6) and Eq. (7), respectively, $\|\bullet\|$ is 2-norm.

Eq. (6) is given as follows

$$
\begin{aligned}
& \nabla F_{\max }(Y)=|\min | \frac{\partial F\left(y_{1}\right)}{\partial y_{1}^{1}}, \frac{\partial F\left(y_{1}\right)}{\partial y_{1}^{2}}, \frac{\partial F\left(y_{1}\right)}{\partial y_{1}^{3}} \mid, \\
& \cdots, \min \left|\frac{\partial F\left(y_{n}\right)}{\partial y_{n}^{1}}, \frac{\partial F\left(y_{n}\right)}{\partial y_{n}^{2}}, \frac{\partial F\left(y_{n}\right)}{\partial y_{n}^{3}}\right|
\end{aligned}
$$

where $Y=\left[y_{1}, \ldots y_{2}\right], \quad y_{1}^{i}(i=1,2,3)$ is $i$ th component of $y_{1}$. If the structure is plane structure, then the third component can be omitted.

Eq. (7) is given as follows

$$
\begin{aligned}
& \nabla F_{\min }(Z)=|\min | \frac{\partial F\left(z_{1}\right)}{\partial z_{1}^{1}}, \frac{\partial F\left(z_{1}\right)}{\partial z_{1}^{2}}, \frac{\partial F\left(z_{1}\right)}{\partial z_{1}^{3}} \mid, \\
& \cdots, \min \left|\frac{\partial F\left(z_{n}\right)}{\partial z_{n}^{1}}, \frac{\partial F\left(z_{n}\right)}{\partial z_{n}^{2}}, \frac{\partial F\left(z_{n}\right)}{\partial z_{n}^{3}}\right| \mid
\end{aligned}
$$

where $Z=\left[z_{1}, \ldots z_{2}\right], \quad z_{1}^{i}(i=1,2,3)$ is $i$ th component of $z_{1}$.

On the other hand, if the LSF is the implicit function, the new FF is shown in Eq. (4). However, the gradient is difficult to be determined. Therefore, the gradient is substituted by one order difference as follows.

$$
\begin{aligned}
& \nabla F(X)=F\left(X^{b}\right)-F\left(X^{a}\right) \\
& \nabla F_{\max }(Y)=\max \left\{F\left(y_{1}^{b}\right)-\right. \\
& \left.F\left(y_{1}^{a}\right), \cdots, F\left(y_{n}^{b}\right)-F\left(y_{n}^{a}\right)\right\} \\
& \nabla F_{\min }(Z)=\min \left\{F\left(z_{1}^{b}\right)\right. \\
& \left.-F\left(z_{1}^{a}\right), \cdots, F\left(z_{n}^{b}\right)-F\left(z_{n}^{a}\right)\right\}
\end{aligned}
$$

where ${ }^{b}$ is the paternal chromosome and ${ }^{a}$ is the progeny chromosome.

The procedure for the proposed method with incorporation of improved fitness GA into the RSM for structural reliability estimation is given as follows. Table 1 Comparison of results for Example 1. 


\begin{tabular}{cccc}
\hline \hline & RSM-MLS & RSM & IGA-RSM \\
\hline $\begin{array}{c}\text { The structural } \\
\text { reliability index } \\
\text { Error }\end{array}$ & 2.7100 & 2.7112 & 2.7111 \\
\hline \multicolumn{4}{c}{ The largest failure point } \\
\hline$u_{1}^{*}$ & -2.5411 & -2.5725 & -2.5722 \\
$u_{2}^{*}$ & 0.9417 & 0.8562 & 0.8958 \\
\hline $\begin{array}{c}\text { Computational } \\
\text { time (s) }\end{array}$ & 50 & 39 & 10 \\
\hline
\end{tabular}

Step 1. The LSF is determined.

Step 2. The $n+1$ design testing points are selected in the variable space, while $f=3$ ( $\mathrm{Li}$ and Chen 2013).

Step 3. The coefficients of Eq. (1) are computed.

Step 4. The maximum failure point of the response surface function $g(\boldsymbol{x})$ is computed by using the first order second moment method.

Step 5. The maximum failure point is given as $\boldsymbol{X}$, the new response surface function is established by using Eq. (1) and Eq. (3) whereas the new FF is established by using Eq. (4).

Step 6. $\nabla F_{\max }(Y)$ and $\nabla F_{\min }(Z)$ are computed, if

$$
\left|\nabla F_{\max }(Y)-\nabla F_{\text {min }}(Z)\right|<\varepsilon^{\prime}
$$

Then $X$ is located as the new central point.

The $2 n+1$ design testing points are selected in the influence domain of the point while $f=1$ ( $\mathrm{Li}$ and Chen 2013).

Step 7. The $2 n+1$ design testing points are substituted into Eq. (1). The coefficients of the second order response surface function $g(\boldsymbol{x})$ are determined.

Step 8. The new surface function is determined. The maximum failure point $X_{D}^{k}$ and the structural reliability index $\beta^{k}$ are computed by using the first order second moment method, where $k$ is the number of the iteration.

Step 9. The Step 5 to Step 8 are repeated until Eq. (12) is satisfied.

$$
\frac{\left\|X_{D}^{k+1}-X_{D}^{k}\right\|}{\left\|X_{D}^{k+1}\right\|}<\varepsilon
$$

where $\varepsilon$ is the required accuracy.

\section{Examples}

Example 1. The example in Rajashekhar and Ellingwood (1993) is used in this paper to verify the proposed method. The LSF is given as follows.

$$
\begin{aligned}
& g(\mathbf{u})=\exp \left[0.4\left(u_{1}+2\right)+6.2\right] \\
& -\exp \left(0.3 u_{2}+5\right)-200
\end{aligned}
$$

where $u_{1}$ and $u_{2}$ are two independent random variables which are considered to obey the standard normal distribution.

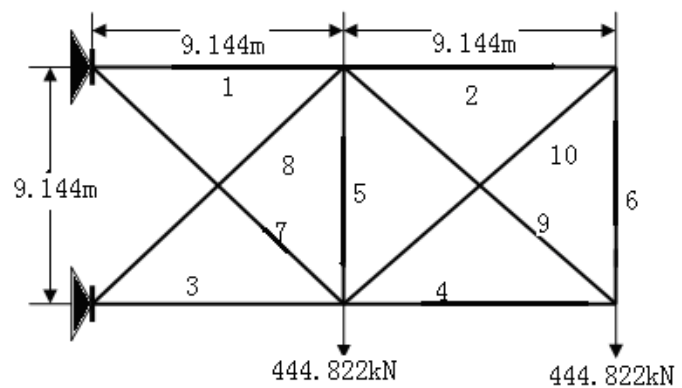

Fig. 1 10-bar truss structure

Table 2 Comparison of results for Example 2

\begin{tabular}{cccc}
\hline \hline & RSM-MLS & RSM & IGA-RSM \\
\hline Reliability index & 4.8083 & 4.8089 & 4.8085 \\
The largest & 7.6658, & 7.6636, & 7.6651, \\
failure points & 9.9949 & 9.9949 & 9.9949 \\
& 9.7302, & 9.7362, & 9.7300, \\
& 10.0075 & 10.0075 & 10.0075 \\
& 10.0350, & 10.0346, & 10.0354, \\
& 9.9949 & 9.9949 & 9.9949 \\
& 9.5916, & 9.5986, & 9.5928, \\
& 10.2997 & 10.2946 & 10.5981 \\
& 10.0212, & 10.0209, & 10.0212, \\
& 9.9855 & 9.9857 & 909855 \\
\hline Computational & \multirow{2}{*}{300} & 240 & 109 \\
\hline
\end{tabular}

The comparison of the results based on RSM using moving least squares (RSM-MLS) (Kang et al. 2010), classical RSM and improved GA for RSM (IGA-RSM) is shown in Table 1. The classical RSM is used as a benchmark method for comparison. It is shown from Table 1 that the computed result obtained by IGA-RSM is more accurate than RSM-MLS, while the number of iteration for IGA-RSM is the lowest among the 3 methods. The size of chromosome population is 10 , cross rate is 0.3 and variation rate is 0.1 . Table 1 gives the method. It is clear that the proposed method is superior in the calculation of structural reliability.

Example 2. A ten-bar truss structure is shown in Figure 1. It is widely used as an example to illustrate structural optimization design and reliability estimation. Its LSF is an implicit function with random variables as shown in Eq. (13).

$$
g(\mathbf{A})=\sigma_{a}-|\sigma(\mathbf{A})|
$$

where $A_{i} \sim N(64.52,1.27)\left(\mathrm{cm}^{2}\right)$ and $\sigma_{a}=172.4 \mathrm{MPa}$.

Similarly, the classical RSM is used as a benchmark method for comparison in this example. It is shown from Table 2 that the reliability index and the largest failure point obtained by IGA-RSM is more accurate than RSM-MLS, while the numbers of iteration of IGA-RSM, classical RSM and RSM-MLS are 34, 84 and 90, respectively. It is clear that the number iteration of IGA-RAM method is the lowest. It can be summarized that the IAG-RSM is superior in structural reliability estimation. 


\section{Conclusions}

In this paper, the improved fitness function of response surface function is established by using the improved GA with explicit and implicit limit state functions. The structural reliability index and the largest failure point can be determined using the improved GA to produce the new response surface function based on the required accuracy. It has been shown by two examples that the proposed method is high precision and needs fewer iterations. The method can be easily accessed in the traditional GA. It has been verified that the algorithm is simple, robust and fast computation.

\section{Acknowledgments}

The work described in this paper was supported in part by a research grant from the Foundation from the Excellent Researcher of Bijie University (G2013017, 2015003), the Science technology Foundation of Guizhou, China (Qian Jiao Ke KY (2014) 226, Qian Jiao Ke KY (2014) 238,), the project of Guizhou province experiment demonstration teaching center, Bijie Key Laborator for New Energy Equipment, Guizhou Province University Coal Chemical Process Equipment and Control Innovation Talent Team (Qian Jiao He Talent Team Zi [2015]73), the Science technology Foundation of Guizhou, China (Qian Ke He HL [2014] 7529) and the Key Laboratory of new energy equipment of Bijie.

\section{References}

Bai, Y.C., Han, X., Jiang, C. and Bi, R.G. (2014), “A responsesurface-based structural reliability analysis method by using non-probability convex model", Appl. Math. Model., 38(15-16), 3834-3847.

Basaga, H.B., Bayraktar, A. and Kaymaz, I. (2012), “An improved response surface method for reliability analysis of structures", Struct. Eng. Mech., 42(2), 175-189.

Ellingwood, B. and Galambos, T.V. (1982), "Probability-based criteria for structural design", Struct. Saf., 1(1), 15-26.

Fang, Y., Chen, J. and Tee, K.F. (2013), "Analysis of structural dynamic reliability based on the probability density evolution method", Struct. Eng. Mech., 45(2), 201-209.

Fang, Y., Wen, L. and Tee, K.F. (2014), "Reliability analysis of repairable k-out-n system from time response under several times stochastic shocks", Smart Struct. Syst., 14(4), 559-567.

Fang, Y., Xiong, J. and Tee, K.F. (2015), “Time-variant structural fuzzy reliability analysis under stochastic loads applied several times", Struct. Eng. Mech., 55(3), 525-534.

He, X. and Liang, J. (2001), "Genetic algorithm using gradients of object function", J. Softw., 12(7), 981-986.

Jiang, C., Lu, G.Y., Han, X. and Liu, L.X. (2012), "A new reliability analysis method for uncertain structures with random and interval variables", Int. J. Mech. Mater. Des., 8(2), 169-182.

Kang, S.C., Koh, H.M. and Choo, J.F. (2010), "An efficient response surface method using moving least squares approximation for structural reliability analysis", Prob. Eng. Mech., 25(4), 365-371.

Khan, L.R. and Tee, K.F. (2016), "Risk-cost optimization of buried pipelines using subset simulation", J. Infrastr. Syst., ASCE,
22(2), 04016001

Kim, C. (2008), "Reliability-based design optimization using response surface method with prediction interval estimation", $J$. Mech. Des., 130(12), 1786-1787

Li, S. and Chen, J. (2013), "A new improved response surface method for structural reliability computing", J. Central South Univ., 44(5), 1837-1841.

Liu, P.L. and Kiureghian, A.D. (1991), "Optimization algorithms for structural reliability”, Struct. Saf., 9(3), 161-177.

Mahmoodian, M., Alani, A.M. and Tee, K.F. (2012), "Stochastic failure analysis of the gusset plates in the Mississippi river bridge", Int. J. Foren. Eng., 1(2), 153-166.

Michhalewicz, Z. (1994), Genetic Algorithm Data Structure Evolution Program, Springer-Verlag, New York.

Ponnambalam, S.G. (2014), "An effective hybrid cuckoo search and genetic algorithm for constrained engineering design optimization”, Eng. Optim., 46(10), 1331-1351.

Rajashekhar, M.R. and Ellingwood, B.R. (1993), "A new look at the response surface approach for reliability analysis", Struct. Saf., 12(3), 5-20.

Shao, J., Cheng, H. and Xu, J. (2001), "Application of genetic algorithm in reliability optimization of structure", Build. Sci. Res. Sichuan, 27(2), 4-5.

Su, G., Jiang, J., Yu, B. and Xiao, Y. (2015), “A Gaussian processbased response surface method for structural reliability analysis", Struct. Eng. Mech., 56(4), 549-567.

Tee, K.F., Khan, L.R. and Coolen-Maturi, T. (2015), "Application of receiver operating characteristic curve for pipeline reliability analysis", J. Risk Reliab. IMechE, Part O, 229(3), 181-192.

Tee, K.F., Khan, L.R., Chen, H.P. and Alani, A.M. (2014), "Reliability based life cycle cost optimization for underground pipeline networks", Tunnel. Underg. Space Tech., 43, 32-40.

Xuan, G. and Chen, R. (2000), Genetic Algorithms and Engineering Design, Science Press, Beijing.

Yang, P., Liu, J. and Guan, Q. (2002), "An improved genetic algorithm for assembly sequence optimization", Comput. Integrat. Manuf. Syst., 8(6), 467-471. 